Research Article

\title{
Experimental Investigation on Mechanical Properties of In Situ Cemented Paste Backfill Containing Coal Gangue and Fly Ash
}

\author{
Xinguo Zhang (iD) and Shichuan Zhang $(D)$ \\ State Key Laboratory of Mining Disaster Prevention and Control, Shandong University of Science and Technology, \\ Qingdao, China \\ Correspondence should be addressed to Shichuan Zhang; 373260186@qq.com
}

Received 18 September 2019; Accepted 3 January 2020; Published 11 February 2020

Academic Editor: Emilio Bastidas-Arteaga

Copyright (c) 2020 Xinguo Zhang and Shichuan Zhang. This is an open access article distributed under the Creative Commons Attribution License, which permits unrestricted use, distribution, and reproduction in any medium, provided the original work is properly cited.

\begin{abstract}
Cemented paste backfill containing coal gangue and fly ash (CGFACPB) is an emerging backfill technique for coal mines that allows environmentally hazardous coal gangue and fly ash to be reused in the underground goaf. Meanwhile, CGFACPB can provide an efficient ground support and reduce the surface subsidence. Due to the difference of consolidation environment between the laboratory and the field, the mechanical properties of the cemented paste backfill vary significantly. In this paper, the core specimens were collected from an underground coal mine where the CGFACPB was used for coal mining, and the mechanical properties of the collected specimens were investigated. The cores were obtained from the underground coal mine, and then the standard cylinders or discs were prepared in laboratory. The uniaxial compressive strength (UCS), Young's modulus, and Poisson's ratio were determined by the compression tests, and the tensile strength was achieved by the Brazilian test. Then the internal friction angle and cohesion were calculated using the improved Mohr-Coulomb strength criterion. The results showed the development of UCS can be divided into four stages, and the final long-term stable value was about 5.1 MPa. The development of Young's modulus had similar trend. Young's modulus had a range from $550 \mathrm{MPa}$ to $750 \mathrm{MPa}$ and the mean value of $675 \mathrm{MPa}$. Poisson's ratio gradually increased with the underground curing duration and eventually approached the stable value of 0.18 . The failure type of compression samples was mainly single-sided shear failure. The development of tensile strength can be divided into two stages, and the stable value of the tensile strength was about $1.05 \mathrm{MPa}$. The development of cohesion can be divided into four stages, and the stable value was about $1.75 \mathrm{MPa}$. The stable value of the internal friction angle was about $25^{\circ}$. This study can provide significant references for not only the long-term stability evaluation of CGFACPB in the field but also the design of optimal recipe of the cemented paste backfill (CPB).
\end{abstract}

\section{Introduction}

Cemented paste backfill containing coal gangue and fly ash (CGFACPB) is an emerging backfill technique in the coal mines in China. The cemented paste backfill can not only dispose of solid waste (mainly coal gangue and fly ash) but also significantly improve the recovery ratio of coal resources and reduce the surface subsidence [1-3]. In the deep underground coal mines in China, CGFACPB is used to replace the residual strip coal pillar; however, the in situ supporting performance of CGFACPB has been a major concern $[1,2]$. The consolidation process of CGFACPB occurs in the complicated underground curing and stress environment. Under the high overlying strata stress, CGFACPB material could collapse or experience an excessive deformation, which could not only affect the mining operations but also cause the serious damages to surface villages [3-6]. CPB is a widely used backfilling method in mining that can significantly improve stope stability in the metal mines or reduce the surface subsidence in the underground coal mines $[1,7]$. In general, CPB materials are produced by mixing solid waste (e.g., coal gangue and tailings), a single or composite hydraulic binder (e.g., Portland cement or slag cement), and water. The CPB materials have a high slump slurry (generally $18-25 \mathrm{~cm}$ ) $[1-3,6]$ and are transported into the underground stope by 
the pipeline system. The main properties of the CPB material include low binder content (3-7\% based on dry weight) and a low uniaxial compressive strength (UCS), i.e., in the range of $0.5 \mathrm{MPa}$ to $5 \mathrm{MPa}[4,6,7]$.

At present, most of studies have been focused on metal mines (e.g., gold, iron, and copper), in which the primary materials were tailing, hydraulic binder, and water, defined as TCPB (tailing cemented paste backfill). Yilmaz et al. [8] investigated the effect of curing time of TCPB with different types of binder and content on the one-dimensional consolidation characteristics using an improved laboratory apparatus. Ghirian and Fall [9] studied the behaviors in the thermal, hydraulic, mechanical, and chemical processes in 1 , 3, and 7 days. Cihangir et al. [10] discussed the effect of activator type, concentration, and slag composition on the strength and stabilities of high-sulphide tailing CPB with alkali-activated slag as the binder. Yilmaz et al. [11, 12] analyzed the effect of specimen size, curing time, and stress conditions on the strength and hydromechanical, geotechnical, and geochemical properties using a new laboratory apparatus. Fall et al. [13, 14] investigated the stress-strain behaviors under the uniaxial compression and the conventional triaxial compression at the humidity of approximately $80 \%$ and the temperature of $23 \pm 2^{\circ}$ in the laboratory. Sivakugan et al. [15-18] conducted a stress-strain test under unconfined and confined pressure in the laboratory. Aldhafeeri et al. [19-21] proposed a relationship between the increase of reactivity and the mechanical damage level of $\mathrm{CPB}$ by analyzing the durability and environmental behavior of $\mathrm{CPB}$ in the chemical reactivity. Ercikdi et al. [22] prepared the sulphide tailings $\mathrm{CPB}$ using the granulated marble waste as an additive and the waste bricks as the replacement and additive to ordinary Portland cement. They showed the using of marble waste samples as an additive could significantly enhance the short- and long-term mechanical strength. Koohestani et al. [23, 24] conducted an experiment on the influence of maple-wood sawdust addition to the mechanical and microstructural properties. Koupouli et al. [25] performed an experimental study on the shear strength of CPB-CPB and CPB-rock wall interfaces. In addition, the fictional behaviors of these interfaces were assessed under the short curing durations using a direct shear apparatus. Based on previous studies, the understanding of engineering properties of TCPB has been significantly improved. As an emerging backfill technique in coal mine, CGFACPB has been studied in fewer studies. Wu et al. [26-28] evaluated the thermal, hydraulic, and mechanical performance of CGFACPB using ultrasonic testing. Yin et al. [29] investigated the effect of solid components on the rheological and mechanical properties of CGFACPB.

The main focus of the above studies is the properties of laboratory-prepared $\mathrm{CPB}$ samples using the conventional moulds. However, some studies have indicated that even the $\mathrm{CPB}$ was prepared using the identical recipe under the same curing conditions and the UCS of field-prepared samples was usually 2-4 times higher than that of the laboratoryprepared samples [30-33]. The difference in the UCS of CPB might be due to the difference in the curing and stress conditions in the field and in laboratory. The conditions in the field improved the strength development rate and the ultimate strength. Due to the significant difference in geological mining conditions, backfilling process, and materials between coal mines and metal mines, the aim of this paper is to investigate the mechanical properties of in situ coring CGFACPB in the laboratory. The UCS, Young's modulus, and Poisson's ratio were determined by the uniaxial compressive tests, and the tensile strength was obtained by the Brazilian splitting test. Based on an improved Mohr-Coulomb strength criterion, the internal friction angle and cohesion were calculated. In addition, some of the damage types of CGFACPB were analyzed.

\section{Materials and Methods}

The core samples were collected from Daizhuang coal mine, located in Jining, Shandong Province, China. In this coal mine, approximately $80 \%$ of coal resources are buried under the villages. The thickness of coal seam is about $2.7 \mathrm{~m}$, and the depth of overlying strata is about $500 \mathrm{~m}$. The traditional longwall caved methods can introduce the severe surface subsidence and thus cannot be used to mine the coal seams. Therefore, a longwall strip mining method was adopted in the coal mine. Currently, a total of 49 residual strip coal pillars exist in the coal mine with the total coal reserves of up to 10 million tons. In order to recycle the residual strip coal pillars, backfilling mining technique was introduced to the coal mine. The first field trial region was selected in the 2300 mining district, and the coal pillar between 2302 and 2303 panel was the targeted object, as shown in Figure 1. The dimensions of coal pillar were $110 \mathrm{~m} \times 960 \mathrm{~m}$ (width $\times$ length). The CGFACPB recipe was determined by a material proportioning test in the laboratory. The ratio of cement:fly ash:coal gangue was 1:4:6, and the solid concentration was $74 \%$. Detailed information on the recipe of CGFACPB can be found in our previous study [34].

\subsection{Coring Process and Samples Preparation in the Field.} Due to the prominent difference in the mining and backfilling operations between metal mines and coal mines, there is a significant discrepancy in the backfilling process. For Daizhuang coal mine, the longwall mining method was used. Therefore, there was a serious interference between coal cutting process and backfilling process. CGFACPB material was gradually backfilled into the goaf region as the working face advanced by about $4-5 \mathrm{~m}$. The segregating operation was conducted to separate the working region and the backfilling region. Usually, a backfilling work was conducted for 3 days every time. The dimensions of the backfilled volume were 4 meters of width, 100 meters of length (longwall working face), and 3 meters of height (working face height). After 28 days in the laboratory, CGFACPB material reached the design strength $[1-3,34]$. At least 10 backfilling processes were required, and as a result, a depth of 40 meters borehole needed to be drilled to obtain the cores. The drilling process was carried out by the TXJ-1600 geological drilling rig (Luheng Instrument, Jining, China). The coring position was about 1.2 meters above the floor. Two drilling boreholes \#1 


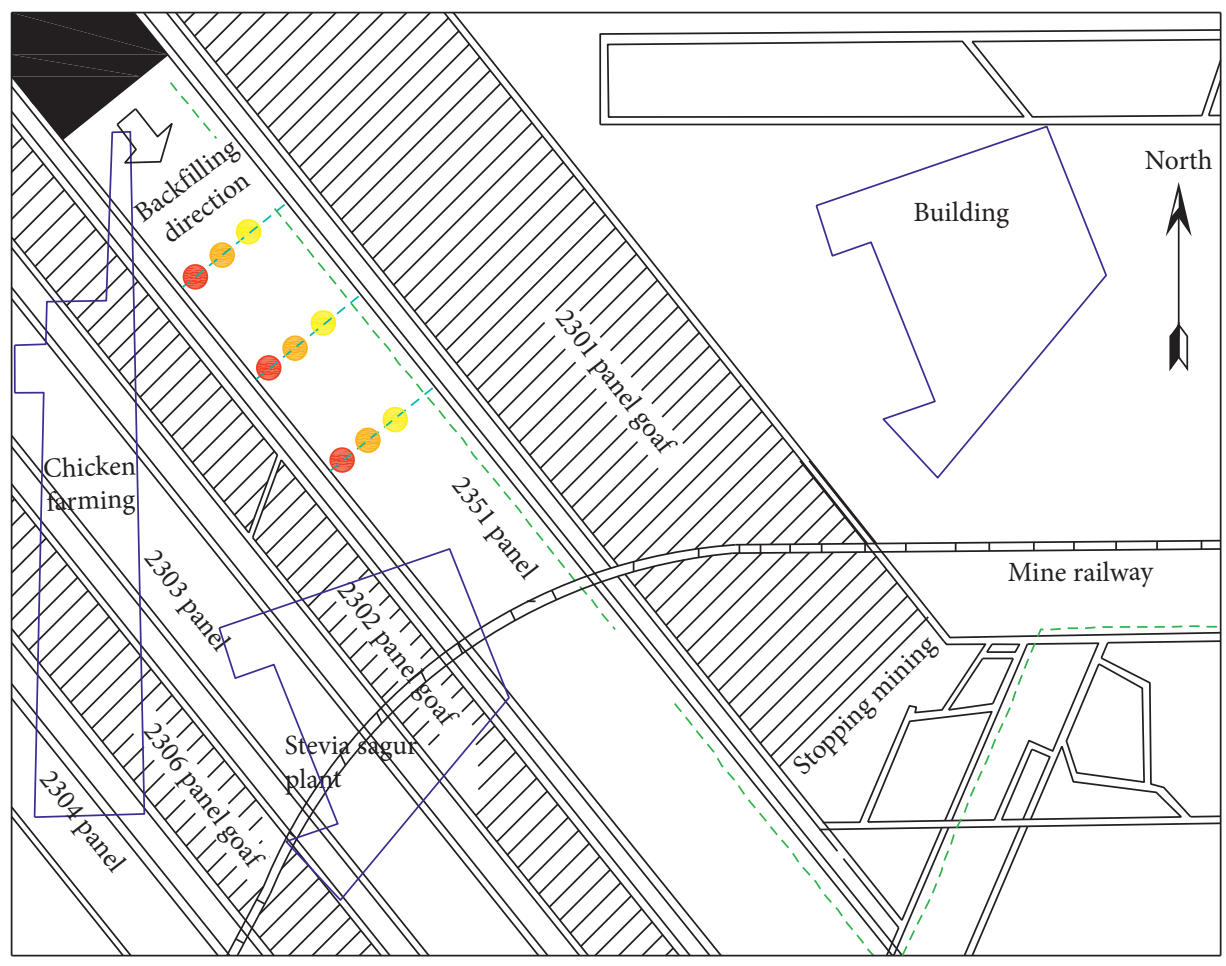

- - Backfilling pipeline

Figure 1: Distribution of coal mine filling working face.

and \#2 were located in the Site I and Site II areas, and their locations are shown in Figure 2. During the coring process, the coring depth and sample numbers were recorded carefully. Plastic wrap was used to seal the samples to prevent the evaporation of water and the damage of sample. Thereafter, the samples were transported to the laboratory and processed to obtain the standard samples indoors $(\Phi 50 \mathrm{~mm} \times 100 \mathrm{~mm}$ compression test; $\Phi 50 \mathrm{~mm} \times 25 \mathrm{~mm}$ tensile test). The testing was conducted immediately. Core samples are shown in Figure 3.

2.2. Testing Methods. Testing was performed in the MTS815.03 rock servo experiment system. The system was controlled by a computer, and the data were collected and recorded automatically. There were three sets of independent servo instruments, which can control axial compression, confining pressure, and pore pressure. The servo valve can respond to the frequency of up to $290 \mathrm{~Hz}$, and stretch sensors can work accurately at the high temperature $\left(200^{\circ} \mathrm{C}\right)$ and oil pressure $(140 \mathrm{MPa})$. The stress-strain curves before and after damage can be measured accurately. For the compression test, the displacement control was adopted, the prepeak loading speed was set to $0.1 \mathrm{~mm} / \mathrm{s}$, and the postpeak loading speed was set to $0.2 \mathrm{~mm} / \mathrm{s}$. The Brazilian disc testing method, proposed by International Society for Rock Mechanics, was used to measure the tensile of CGFACPB samples. In order to reduce the influence of the interface friction on the strength of the sample, the rigid ingot head was used. The MTS815.03 rock servo experiment system, the compression (UCS) test system, and tensile test system are shown in Figures 4 and 5, respectively.

\section{Results and Discussion}

3.1. Compression Test Results. In this study, 232 standard samples were obtained from two drillings. Because the backfill was very fragile, fewer standard samples were obtained. On one hand, the CGFACPB material had a lower strength and lower coring ratio. As a result, it was very difficult to acquire long intact cores. On the other hand, damages occurred during the transportation and laboratory processing. There were 121 cores for drilling \# 1 (Figure 2 Site I) and 111 cores for drilling \#2 (Figure 2 Site II). From boreholes \#1 and \#2, there were 58 and 51 cores used for the compression test, respectively. The UCS, Young's modulus, and Poisson's ratio were obtained by the compression testing. Due to the large discreteness of the testing results, the mean values of samples with the same curing time in the underground were used to evaluate the testing results. Figure 6 illustrates the development of UCS with the underground curing time. The underground backfilling process can be used to interpret the evolution of UCS with the underground curing time and the roof stress environment, as shown in Figure 7. Combined with the process of filling mining, we interpret the USC change process of the filling body.

As we all know, the UCS of CPB obtained from the uniaxial compressive tests is the most important parameter to evaluate the property or recipe value of $\mathrm{CPB}$. From the 


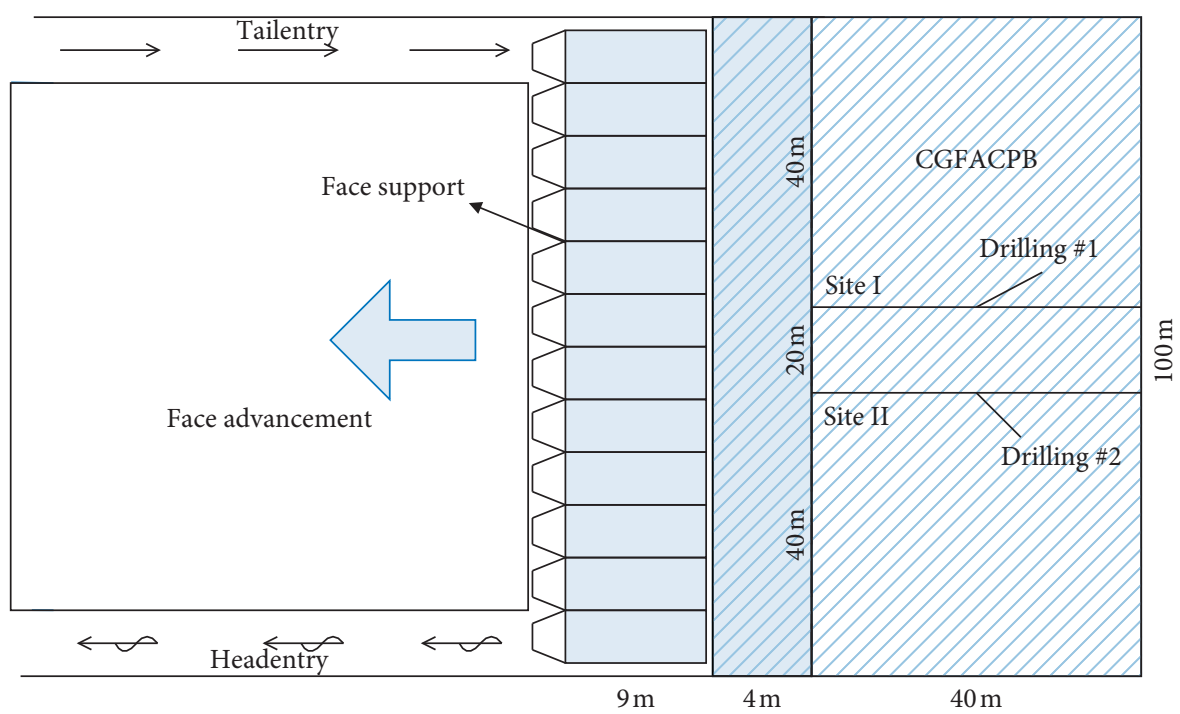

FIgURE 2: Field coring layout plan.
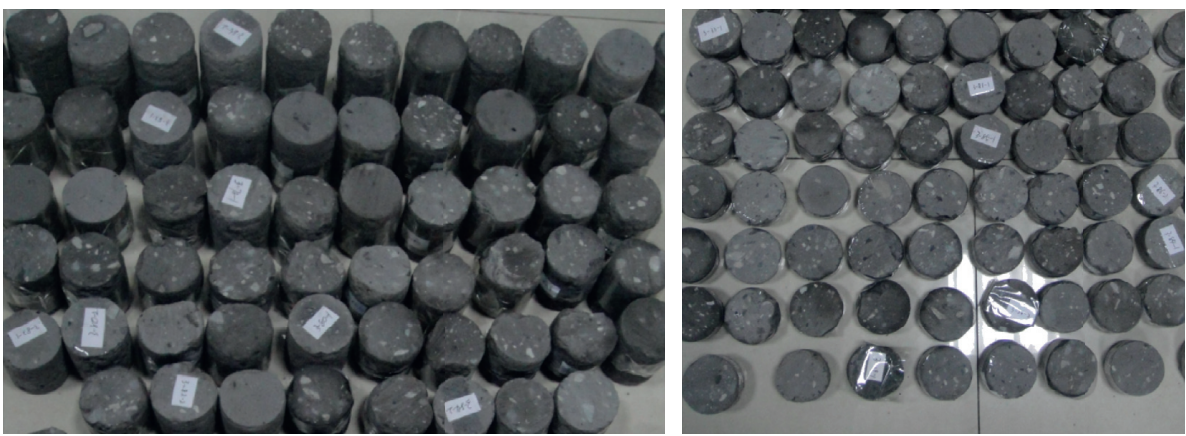

FIgURE 3: Standard samples after cutting.

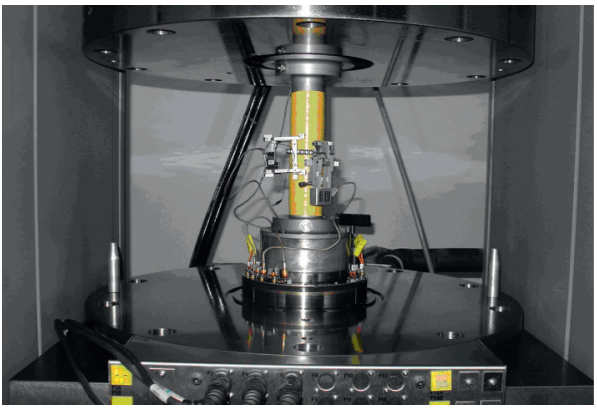

FIGURE 4: UCS test system.

fitting curve of UCS with the underground curing time, it can be observed that the evolution of UCS can be divided into four stages:

(a) Rapid development stage I: in this stage, the consolidation of CGFACP changed from the slurry to the solid, and the initial strength value continuously increased from $0 \mathrm{MPa}$ up to self-standing strength (generally, the curing time is 8 hours in the laboratory because the hydraulic supports move forward with the advancement of working face). In the

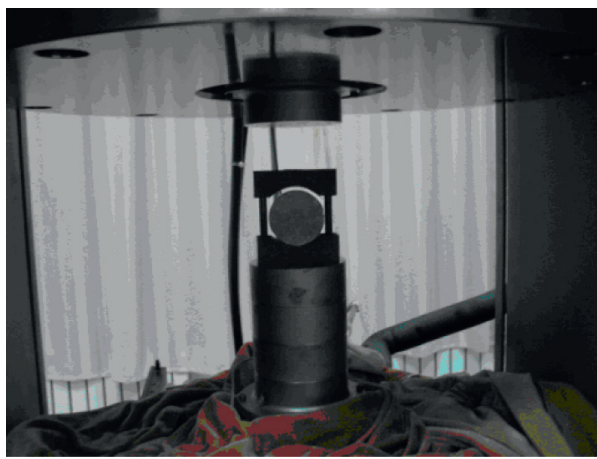

FIgURE 5: Tensile strength test system.

working face, the consolidation conditions of CGFACP mainly included curing condition and stress condition. The curing condition consisted of temperature and humidity of the working face. The stress condition was dependent on the roof strata loading. Generally the curing condition of the first block backfill was different from that of other blocks because one side of the backfill was the working face and the other side was backfill. The first block backfill 


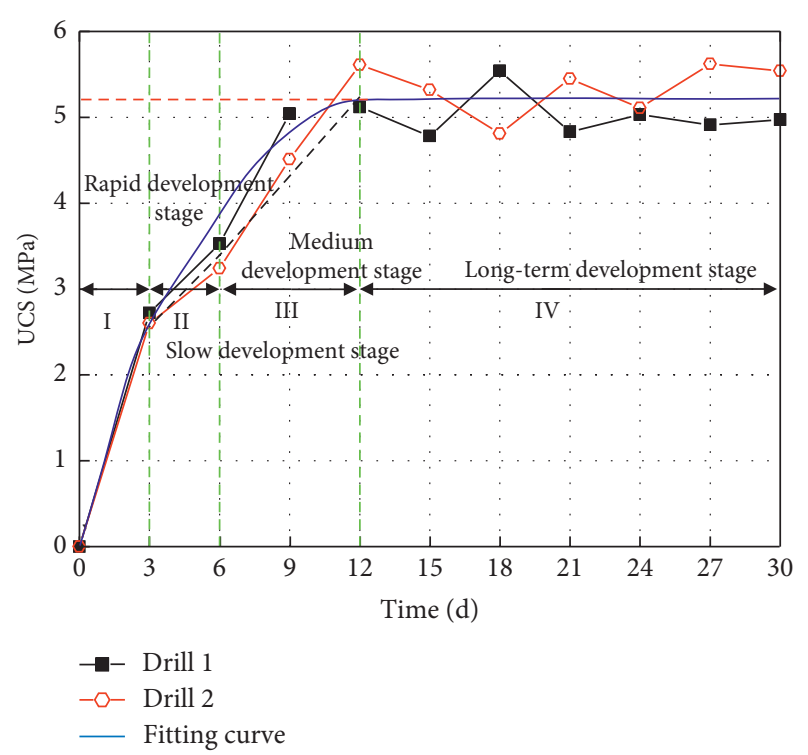

FIGURE 6: Development of UCS with the underground curing time.

was under the cover of face supports and the back backfill; thus the roof stress loading generally was little and often negligible according to the field experience. The value of UCS within three days was increased from $0 \mathrm{MPa}$ to $2.72 \mathrm{MPa}$ in drilling \#1 and to $2.60 \mathrm{MPa}$ in drilling \#2, indicating that the growth velocity was rapid, which can be observed from the fitting curve.

(b) Slow development stage II: when the second block backfill was gradually backfilled into the goaf region, the first block had been cured for three days in the underground. In this stage, the consolidation environment of the first block had changed not only the curing environment but also the stress environment. The curing temperature and humidity of the first block had significant change, and the roof stress was gradually increased. The UCS value was increased from $2.72 \mathrm{MPa}$ to $3.53 \mathrm{MPa}$ in drilling \#1 and from 2.60 MPa to 3.24 MPa in drilling \#2. The growth rate of UCS was relatively lower in the following three days.

(c) Medium development stage III: in this stage, the backfill strength increased continuously. The first backfill had been cured for nine days. The curing temperature and humidity of the first block might have been stable or decreased, while the roof stress applied on the backfill had gradually increased. At this time, the overhead roof distance from the first block to coal mass had been up to approximately 20 meters. The direct roof strata might have been sank, bent, and broken. The UCS value after was from $3.53 \mathrm{MPa}$ to $5.04 \mathrm{MPa}$ in drilling \#1 and from 3.24 MPa to $4.51 \mathrm{MPa}$ in drilling \#2. The growth rate of UCS was relatively gentle.

(d) Gradual or long-term stable stage IV: after twelve days, a stable trend can be observed from the fitting curve. The value of UCS was stable at about 5.1 MPa. In this stage, the surrounding environment of paste had a large change on not only curing environment but also roof stress environment.

Young's modulus is another important index, which reflects the resistance of the material to elastic deformation. The larger Young's modulus indicates a greater stiffness of the material. From the fitting curve in Figure 8, the development trend of Young's modulus was similar to that of the UCS. Thus, the development of Young's modulus can also be divided into four stages. In the first stage, i.e., the rapid development stage, the CGFACPB slurry was changed from fluidity to solidity in the underground curing environment. In the second stage, i.e., the medium development stage, Young's modulus value was increased from the minimum of $475 \mathrm{MPa}$ up to $610 \mathrm{MPa}$ in drilling \#1 and from $175 \mathrm{MPa}$ up to $525 \mathrm{MPa}$ in drilling \#2. The third stage, i.e., slow development stage, lasted for 6 days. From Figure 8 , there is a large discreteness in the value of Young's modulus in both drilling \#1 and drilling \#2. In the fourth stage, i.e., long-term development stage, the relatively stable trend can be observed from the fitting curve. Young's modulus was in the range of $550 \mathrm{MPa}$ to $750 \mathrm{MPa}$. The value of Young's modulus in the fitting curve was $675 \mathrm{MPa}$.

Poisson's ratio is an elastic parameter to characterize the lateral deformation capacity of materials. Poisson's ratio reflects the ratio between the deformation magnitudes of the material in two different directions. Larger Poisson's ratio indicates that the transverse deformation is larger than the longitudinal deformation. Figure 9 shows the trend of Poisson's ratio with the underground curing time. Within the first twelve days, Poisson's ratio was gradually increasing in both drilling \#1 and drilling \#2. Thereafter, the change of Poisson's ratio was not obvious. Poisson's ratio was between 0.17 and 0.19 in drilling \#1 and between 0.19 and 0.28 in drilling \#2. Poisson's ratio was gradually increased in the first 12 days and stable at 0.18 .

Generally, the rock has three failure types under the unconfined or uniaxial compression loading, including $\mathrm{X}$-conjugate oblique shear failure, single slope shear failure, and splitting failure [35]. CGFACPB material was a lowgrade concrete material, which was also called similar rock material. Some typical failures of different samples are shown in Figure 10. From the figure, the failure of the samples was mainly single-sided shear failure.

3.2. Tensile Test Results. In this study, 63 blocks in drilling \# 1 and 60 blocks in drilling \#2 were used in the tensile test. The Brazilian method was used to test the tensile strength of the samples. Figure 11 shows the development of the tensile strength with the underground curing time. The investigation on the tensile strength of the sample can provide significant reference to not only the stability of cavern project but also the slicing backfill mining of thick coal seam. From Figure 11, the development of the tensile strength can be approximately divided into two stages. In the first stage, i.e., the development stage within the first twelve days, the 


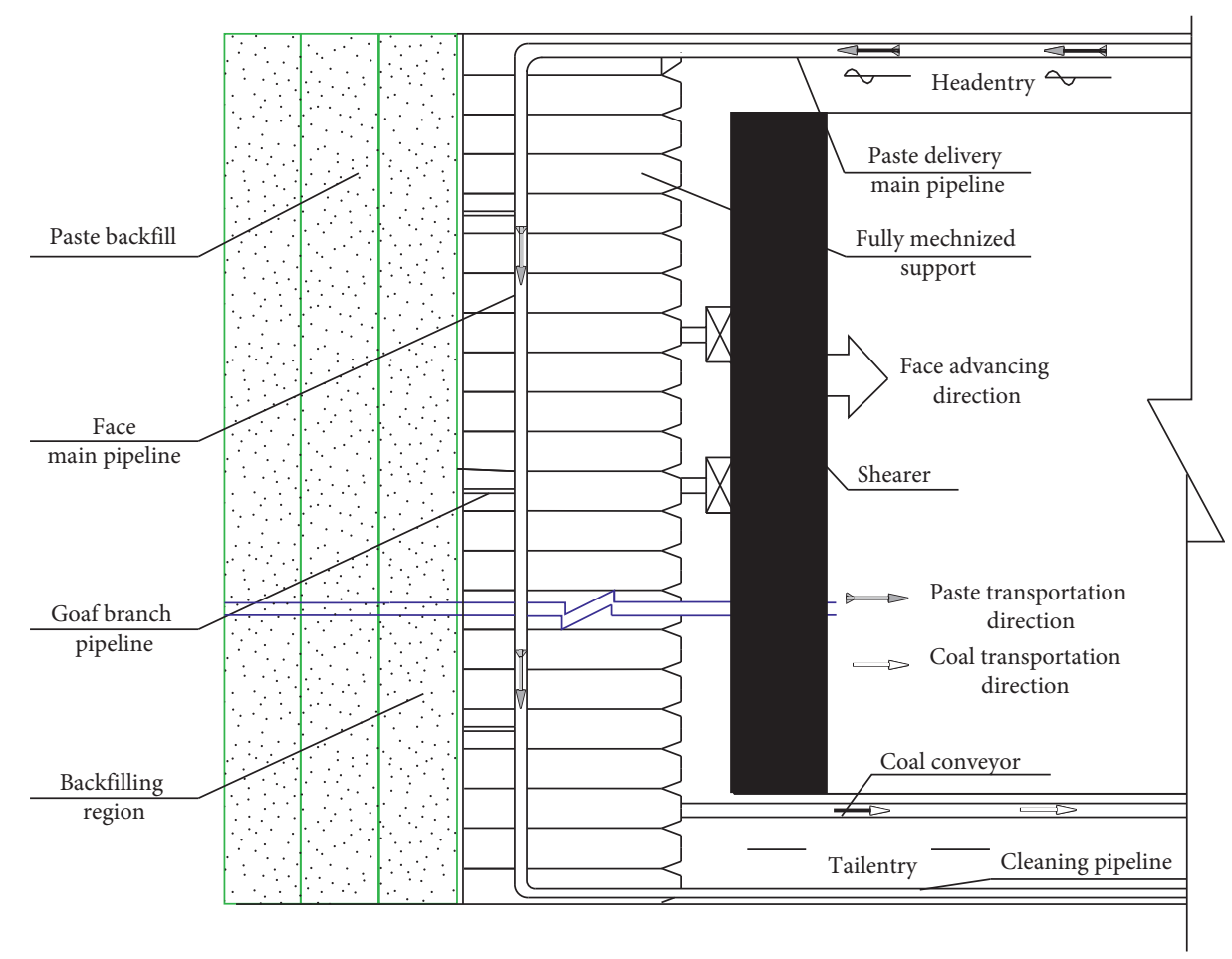

(a)

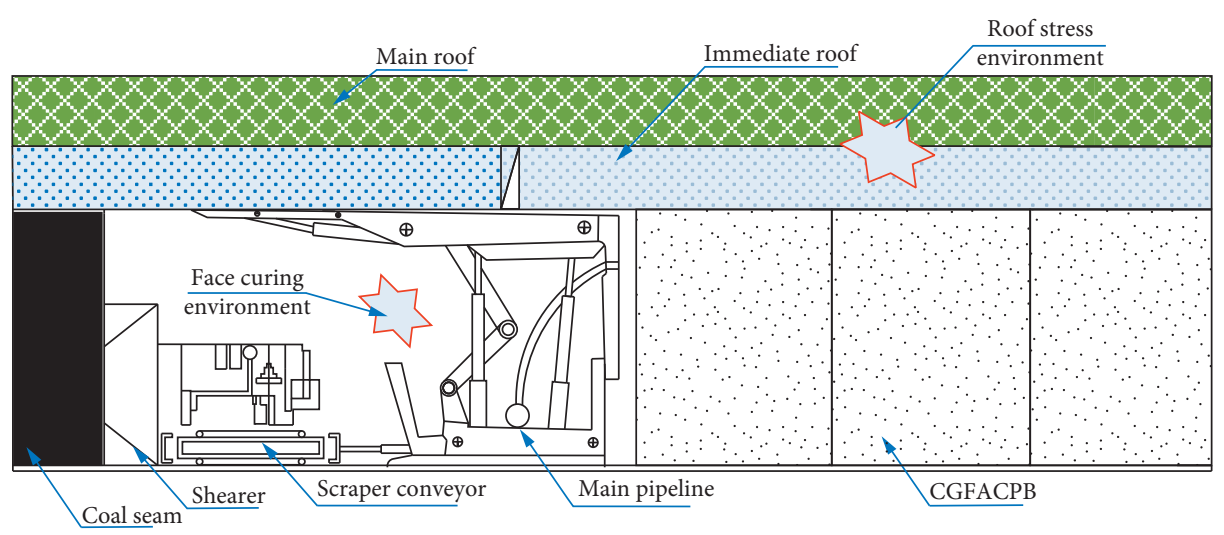

(b)

FIGURE 7: Schematic diagram of longwall backfilling curing and stress environment. (a) Backfilling panel. (b) Cross section.

strength value gradually increased. In the second stage, i.e., gradual development or long-term stable stage, the tensile strength value was in the range of $1.05 \mathrm{MPa}$ to $1.26 \mathrm{MPa}$ for drilling \#1 and in the range of $0.82 \mathrm{MPa}$ to $1.25 \mathrm{MPa}$ for drilling \#2. The value from the fitting curve was about $1.05 \mathrm{MPa}$. Empirically, the tensile strength of rock is $1 / 10-1 /$ 20 of UCS. However, the ratio of the tensile strength value to the obtained UCS value was obviously larger with the underground curing time. The testing results of the tensile strength showed that CGFACPB material had better resistance to shear deformation. Some photographs of the typical damage are shown in Figure 12.
3.3. Cohesion and Internal Friction Angle. In this study, in order to calculate the cohesion and the internal friction angle of CGFACPB, Mohr-Coulomb strength criterion was used $[36,37]$. The equation is as follows:

$$
\tau=c+\sigma \tan \phi
$$

where $\tau$ is the shear stress, $\sigma$ is the normal stress, $c$ is the cohesion, and $\phi$ is the internal friction angle. The Mohr-Coulomb strength criterion can be expressed by the Mohr limit circle, as shown in Figure 13.

$\sigma$ and $\tau$ can be expressed by the principal stress $\sigma_{1}$ and $\sigma_{3}$. The equation is as follows: 


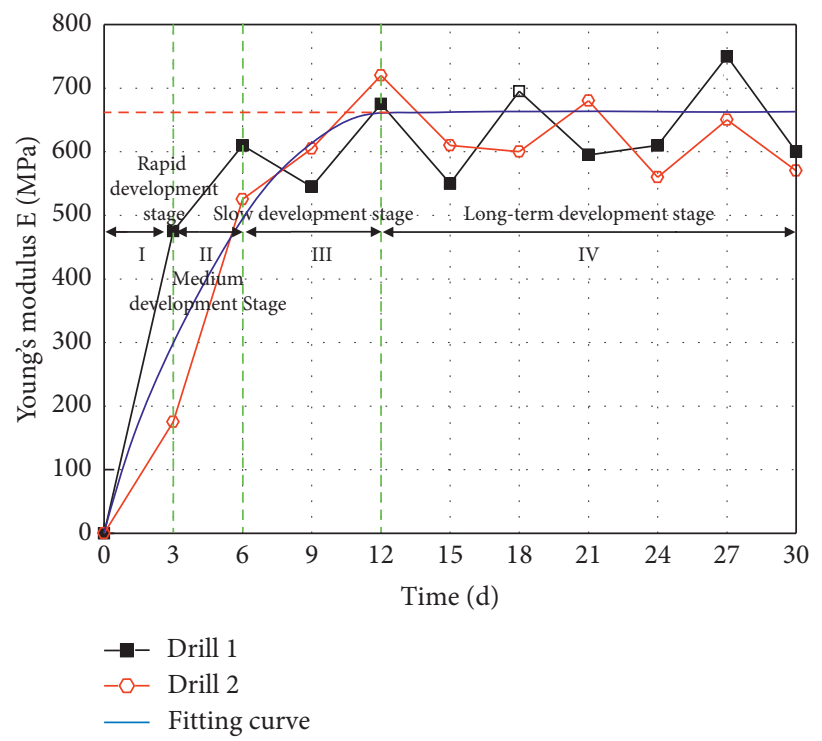

Figure 8: Development of Young's modulus with the underground curing time.

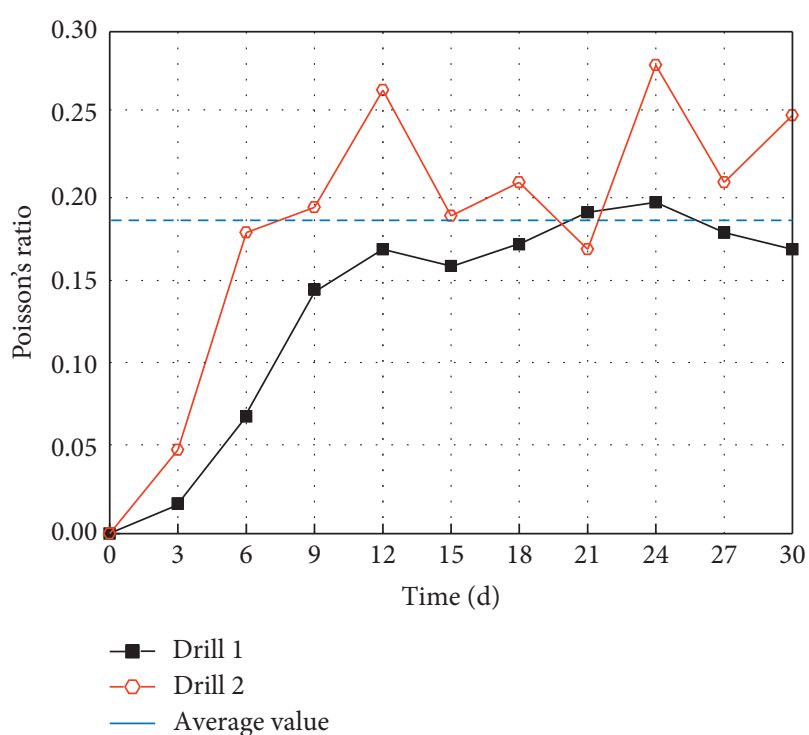

Figure 9: Trend of Poisson's ratio with the underground curing time.

$$
\left.\begin{array}{l}
\sigma=\frac{1}{2}\left(\sigma_{1}+\sigma_{3}\right)+\frac{1}{2}\left(\sigma_{1}-\sigma_{3}\right) \cos 2 \theta \\
\tau=\frac{1}{2}\left(\sigma_{1}-\sigma_{3}\right) \sin 2 \theta \\
2 \theta=\frac{\pi}{2}+\varphi
\end{array}\right\},
$$

where $\theta$ is the broken angle of rock and $\sigma_{3}$ are the principal stresses.

Substituting equation (2) into equation (1), the Mohr-Coulomb strength criterion can be expressed as

$$
\sigma_{1}=\frac{1+\sin \phi}{1-\sin \phi} \sigma_{3}+\frac{2 c \cdot \cos \phi}{1-\sin \phi} .
$$

When $\sigma_{3}=0, \sigma_{1}$ is the $\sigma$ of UCS, then the equation can be written as follows:

$$
\sigma=\frac{2 c \cdot \cos \varphi}{1-\sin \varphi} .
$$

Therefore, the equation of the Mohr-Coulomb strength criterion in the $\sigma_{3}-\sigma_{1}$ coordinate system is as follows:

$$
\sigma_{1}=\sigma_{3} \tan ^{2} \theta+\sigma_{c}
$$

where $\sigma_{c}$ is the UCS.

Substituting $\tan \varphi=f$ and equation (2) to equation (1), the equation can be rewritten as follows:

$$
\tau-f \sigma=\frac{1}{2}\left(\sigma_{1}-\sigma_{3}\right)(\sin 2 \theta-f \cos 2 \theta)-\frac{1}{2} f\left(\sigma_{1}+\sigma_{3}\right) .
$$

Taking the derivative of this equation to $\theta$, the maximum of $\tau-f \sigma$ can be obtained as follows:

$$
\{\tau-f \sigma\}_{\mathrm{mas}}=\frac{1}{2}\left(\sigma_{1}-\sigma_{3}\right) \sqrt{f^{2}+1}-\frac{1}{2} f\left(\sigma_{1}+\sigma_{3}\right) .
$$

If the value of equation (7) is less than $c$, the rock would not break; otherwise, the rock would break. If $\{\tau-f \sigma\}_{\text {mas }}=c$, equation (7) can be written as

$$
2 c=\sigma_{1}\left[\sqrt{f^{2}+1}-f\right]-\sigma_{3}\left[\sqrt{f^{2}+1}+f\right] .
$$

The intensity curve of the Mohr-Coulomb strength criterion in the $\sigma_{3}-\sigma_{1}$ coordinate system is shown in Figure 14.

Thereafter, $\sigma$ can be expressed as follows:

$$
\sigma_{c}=2 c\left[\sqrt{f^{2}+1}+f\right] \text {. }
$$




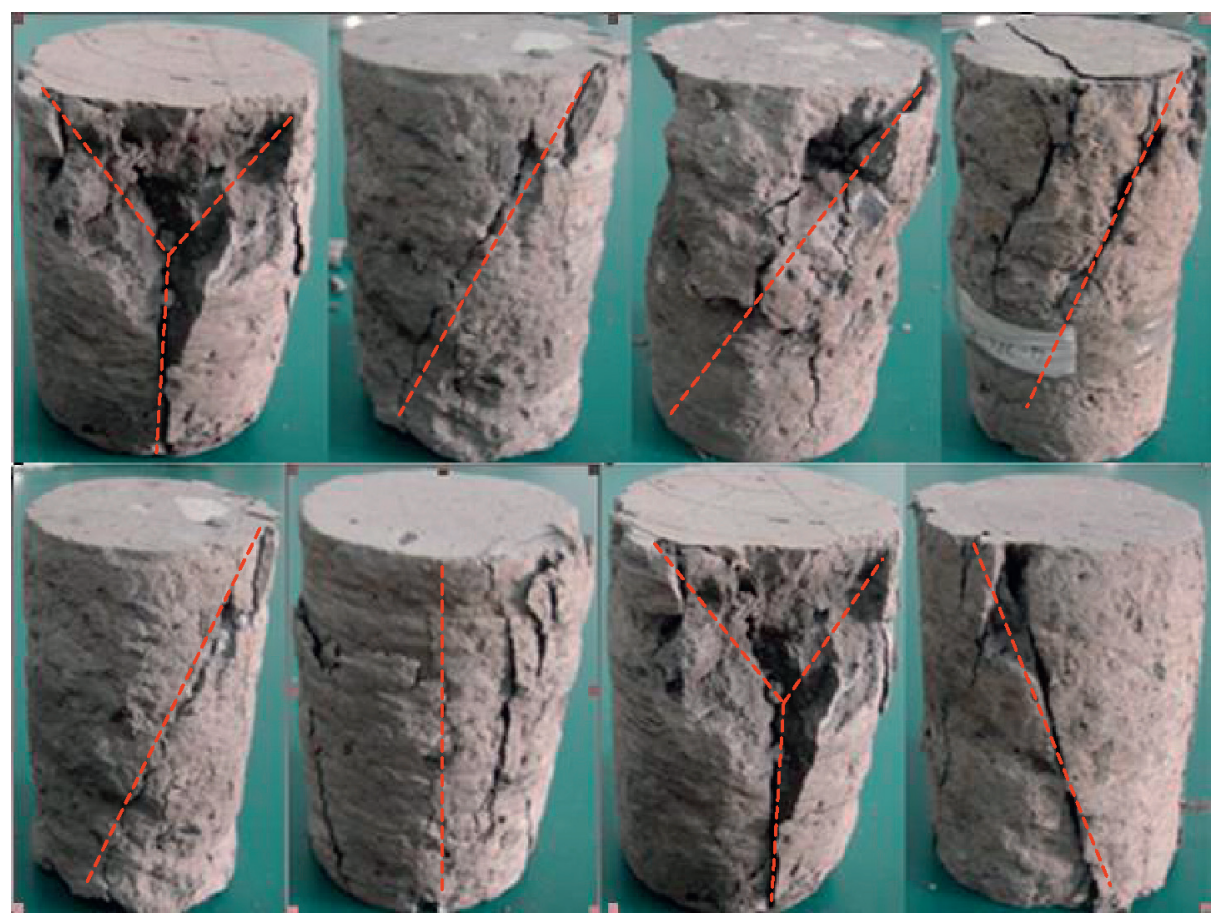

Figure 10: Photographs of damage in the compression samples.

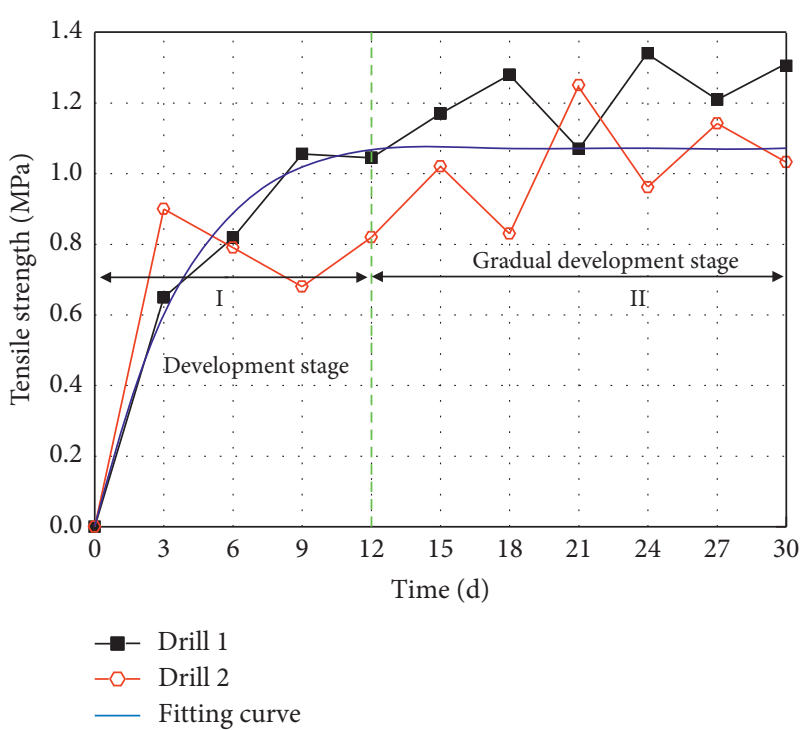

Figure 11: Development of tensile strength with the underground curing time.

The minimum value of $\sigma_{1}$ can be used to determine when the rock is going to break in the maximum limit equilibrium state. If $\sigma>0$ and any $\theta$ value, the following equation is obtained from equation (2):

$$
2 c=\sigma_{1}(1+\cos 2 \theta)+\sigma_{3}(1-\cos 2 \theta) .
$$

Since $\quad \cos 2 \theta=-\left(f / \sqrt{f^{2}+1}\right), \quad 2 \sigma=\sigma_{1}[1-(f /$ $\left.\left.\sqrt{f^{2}+1}\right)\right]+\sigma_{3}\left[1+\left(f / \sqrt{f^{2}}+1\right)\right]$. Since $\sqrt{f^{2}+1}>0$, in the case $\sigma>0$, the following equation can be obtained:

$$
\begin{aligned}
\sigma_{1}\left[\sqrt{f^{2}+1}-f\right]+\sigma_{3}\left[\sqrt{f^{2}+1}+f\right] & >0, \\
\sigma_{1} & >\frac{1}{2} \sigma_{c} .
\end{aligned}
$$

The equation of the Mohr-Coulomb strength criterion in the $\sigma_{3}-\sigma_{1}$ coordinate system can be expressed as follows:

$$
\begin{aligned}
& \sigma_{1}\left[\sqrt{f^{2}+1}-f\right]-\sigma_{3}\left[\sqrt{f^{2}+1}+f\right]=2 c \cdot\left(\sigma_{1}>\frac{1}{2} \sigma_{c}\right) \\
& \sigma_{3}=-\sigma_{1} \cdots\left(\sigma_{1} \leq \frac{1}{2} \sigma_{c}\right)
\end{aligned}
$$

Therefore, when $\sigma_{1}=0, \sigma_{3}=\sigma_{t}<0, \beta$ of the straight line PL can be gained in the following equation: $\operatorname{tg} \beta=2 \sigma_{t} / \sigma_{c}$.

Therefore, the Mohr-Coulomb strength criterion in the $\sigma_{3}-\sigma_{1}$ coordinate system can be determined by the value of UCS and the tensile strength indirectly. The equations of the internal friction angle and the cohesion can be expressed by the value of UCS and the tensile strength, as shown in the following equations:

$$
\begin{aligned}
& \varphi=2 \theta-\frac{\pi}{2}=2 \arctan \sqrt{\frac{1}{\tan \beta}}-\frac{\pi}{2}=2 \arctan \sqrt{\frac{\sigma_{c}}{2 \sigma_{t}}}-\frac{\pi}{2}, \\
& c=\frac{\sigma_{c}}{2 \tan \theta}=\frac{\sigma_{c}}{2} * \sqrt{\tan \beta} .
\end{aligned}
$$

Based on the testing results of UCS and tensile strength, the value of the cohesion and the internal friction angle with 


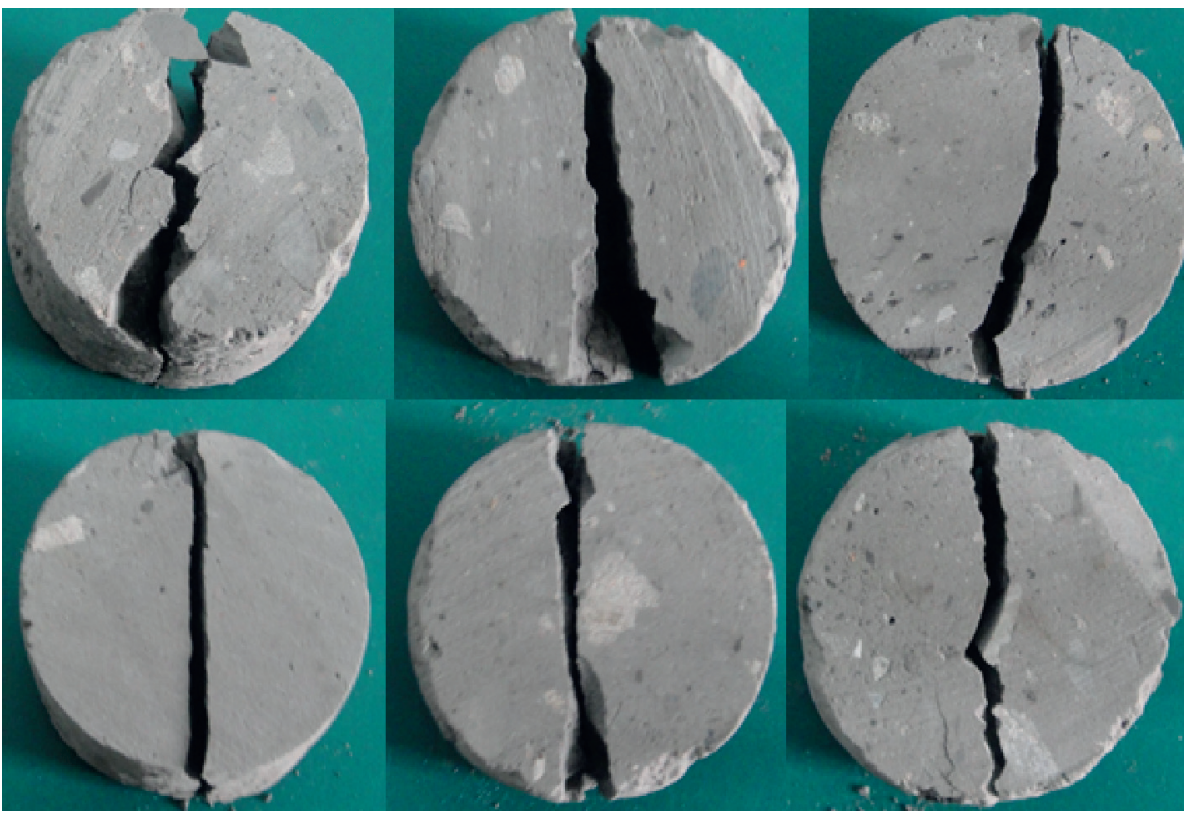

Figure 12: Photographs of some damage in the tensile test.
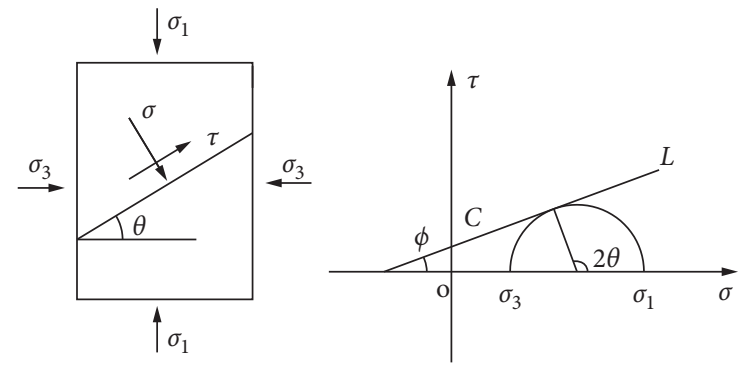

Figure 13: The coulomb criterion in $\sigma-\tau$ coordinates.

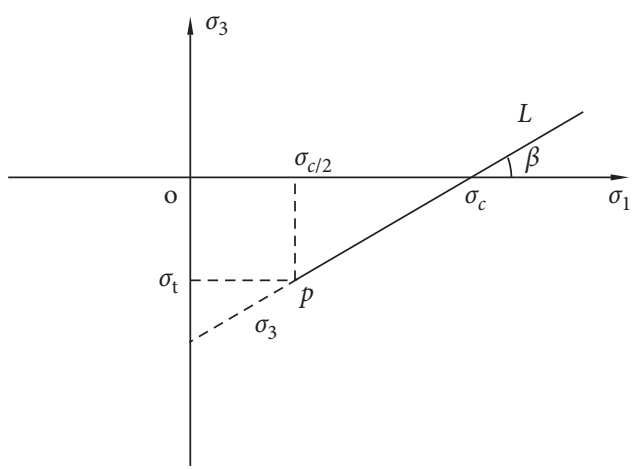

FIGURE 14: The coulomb criterion in the $\sigma_{3}-\sigma_{1}$ coordinate system.

the curing time can be calculated. The trends of the cohesion and the internal friction angle with the curing time are shown in Figures 15 and 16, respectively. The cohesion and the internal friction angle were two important parameters to reflect the resistant capability to the shear strength of rock mass and soil mass. The development of cohesion of CGFACPB material can be divided into four stages. In the first stage, i.e., rapid development stage, within the first three

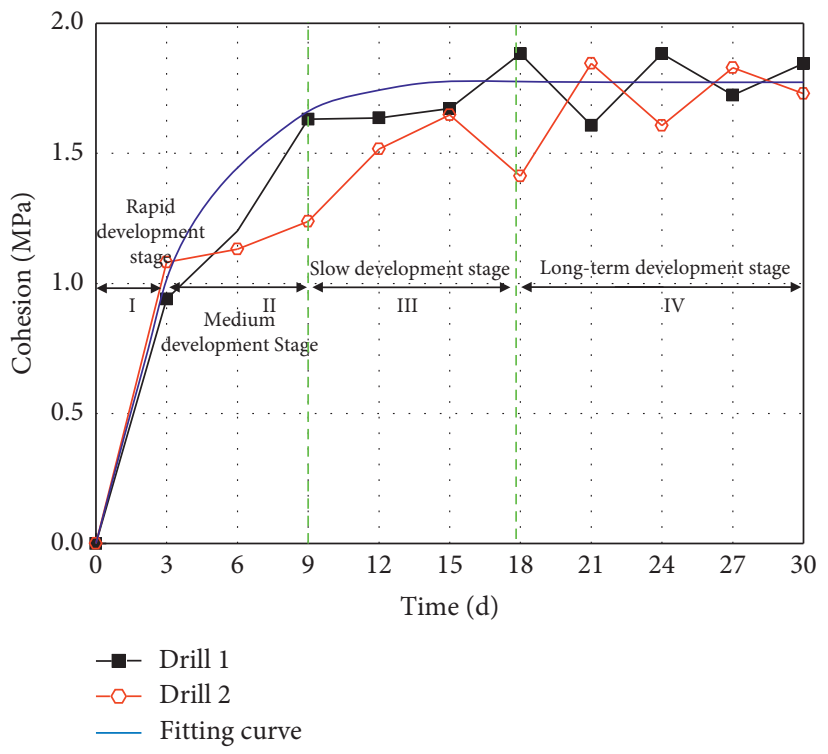

FIGURE 15: Development of cohesion with the underground curing time.

days, the cohesion value was increased from $0 \mathrm{MPa}$ to $0.94 \mathrm{MPa}$ in drilling \#1 and from $0 \mathrm{MPa}$ to $1.08 \mathrm{MPa}$ in drilling \#2. In the second stage, i.e., medium development stage, from the third day to the ninth day, the cohesion value was increased from 1.08 MPa to $1.23 \mathrm{MPa}$ in drilling \#1 and from $0.94 \mathrm{MPa}$ to $1.63 \mathrm{MPa}$ in drilling \#2. The development velocity in the second stage was slightly lower than that of the first stage. In the third stage, i.e., slow development stage, there was a certain discreteness in the value. In the fourth stage, i.e., gradual or long-term development stage, although the values had a large discreteness, a stable value can be observed from the fitting curve. The approached stable value from the fitting value was about $1.75 \mathrm{MPa}$. 


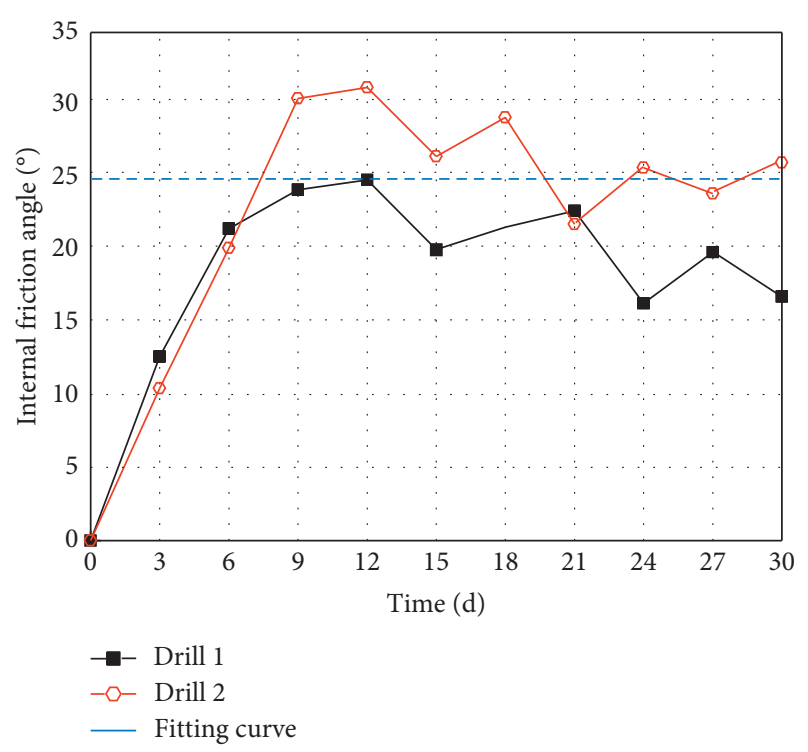

FIGURE 16: Trend of the internal friction angle with the curing time.

The internal friction angle refers to the angle between the positive stress and the internal friction stress, which is formed when the rock approaches the limit equilibrium. As an index of the shear strength, the internal friction angle reflects the friction property of rock and is an important engineering design parameter. From the results in Figure 16, according to the improved Mohr-Coulomb strength criterion, the internal friction angle value had a large discreteness, and the internal friction angle value was stable at about $25^{\circ}$.

\section{Conclusions}

In this paper, an experimental investigation was conducted on the mechanical parameters of CGFACPB cored from an underground coal mine. The main results are summarized as follows:

(1) The ingredients of CGFACPB materials were analyzed, and the production process was introduced. The primary ingredients of CGFACPB materials include coal gangue, fly ash, OPC, and water. The field recipe of CGFACPB was described as follows: the ratio of OPC: fly ash: coal gangue was $1: 4: 6$, and the solid concentration was $74 \%$. Simultaneously the in situ coring layout plan and test procedures were provided.

(2) Compression test showed that the development of UCS can be divided into four stages, i.e., rapid development stage, slow development stage, medium development stage, and gradual stable stage. The long-term stable value of UCS was about 5.1 MPa. The development of Young's modulus had similar growing trend. The range of Young's modulus was between $550 \mathrm{MPa}$ and $750 \mathrm{MPa}$. According to the fitting curve, the stable value was $675 \mathrm{MPa}$. The value of Poisson's ratio was gradually increased within the first twelve days in both drilling \#1 and drilling \#2.
After the increasing stage, Poisson's ratio approached the stable value of 0.18 . The failure type of uniaxial compression samples was mainly singlesided shear failure.

(3) Brazilian test results showed that the development of tensile strength can be divided into two stages, i.e., development stage and gradual stable stage. The range of tensile strength was between $0.82 \mathrm{MPa}$ and $1.26 \mathrm{MPa}$, and the mean value was about $1.05 \mathrm{MPa}$. The results indicated that CGFACPB material had better resistance to shear deformation.

(4) By an improved Mohr-Coulomb strength criterion, the cohesion and internal friction angle were calculated. The development of cohesion of CGFACPB material can be divided into four stages, i.e., rapid development stage, medium development stage, slow development stage, and long-term development stages. The stable value of cohesion was about 1.75 MPa. The internal friction angle value had a large discreteness, and the stable value was about $25^{\circ}$.

This study can provide significant reference for not only the evaluation of the supporting properties of CGFACPB in the field but also the design of the optimal $\mathrm{CPB}$ recipe. However, the field CGFACPB material is under the threedimensional stress, and there may be a larger difference in the results of mechanical properties from the unconfined compression test and the triaxial compression test. Currently, it is very difficult to measure the confining pressure of CGFACPB on the site. The next study may be focused on the investigation on the mechanical properties of $\mathrm{CPB}$ under triaxial loading test.

\section{Data Availability}

The data used to support the findings of this study are available from the corresponding author upon request.

\section{Conflicts of Interest}

The authors declare no conflicts of interest.

\section{Authors' Contributions}

Xinguo Zhang proposed the idea and drafted the manuscript. Shichuan Zhang analyzed the ingredients of CPB, conducted the indoor rock testing, and prepared the manuscript.

\section{Acknowledgments}

This study was supported by the National Natural Science Foundation of China (Nos. 51574159, 51428401, and 51974173), Shandong Province Natural Science Foundation (No. ZR2014EEM001), Shandong Province Key R\&D Program (Nos. 2018GSF116002 and 2019GSF111024), and SDUST Research Fund, Taishan Scholar Talent Support Plan for Advantaged and Unique Discipline Areas. 


\section{References}

[1] X.-m. Wang, B. Zhao, C.-s. Zhang, and Q.-1. Zhang, "Pastelike self-flowing transportation backfilling technology based on coal gangue," Mining Science and Technology (China), vol. 19, no. 2, pp. 137-143, 2009.

[2] S.-h. Yin, Y.-j. Shao, A.-x. Wu, Y.-m. Wang, and Z.-y. Gao, "Texture features analysis on micro-structure of paste backfill based on image analysis technology," Journal of Central South University, vol. 25, no. 10, pp. 2360-2372, 2018.

[3] D. Wu, G. Sun, and Y. Liu, "Modeling the thermo-hydrochemical behavior of cemented coal gangue-fly ash backfill," Construction and Building Materials, vol. 111, pp. 522-528, 2016.

[4] D. R. Tesarik, J. B. Seymour, and T. R. Yanske, "Long-term stability of a backfilled room-and-pillar test section at the Buick mine, Missouri, USA," International Journal of Rock Mechanics and Mining Sciences, vol. 46, no. 7, pp. 1182-1196, 2009.

[5] R. Karim, G. M. Simangunsong, B. Sulistianto, and A. Lopulalan, "Stability analysis of paste fill as stope wall using analytical method and numerical modeling in TheKencana underground gold mining with long hole stope method," Procedia Earth and Planetary Science, vol. 6, pp. 474-484, 2013.

[6] V. Lilkov, I. Rostovsky, O. Petrov, Y. Tzvetanova, and P. Savov, "Long term study of hardened cement pastes containing silica fume and fly ash," Construction and Building Materials, vol. 60, pp. 48-56, 2014.

[7] Y. Xu, Q. Chang, H. Zhou, Z. Cao, X. Li, and J. Chen, "Movement and deformation laws of the overlying strata in paste filling stope," Mining Science and Technology (China), vol. 21, no. 6, pp. 863-868, 2011.

[8] E. Yilmaz, T. Belem, B. Bussière, M. Mbonimpa, and M. Benzaazoua, "Curing time effect on consolidation behaviour of cemented paste backfill containing different cement types and contents," Construction and Building Materials, vol. 75, pp. 99-111, 2015.

[9] A. Ghirian and M. Fall, "Coupled behavior of cemented paste backfill at early ages," Geotechnical and Geological Engineering, vol. 33, no. 5, pp. 1141-1166, 2015.

[10] F. Cihangir, B. Ercikdi, A. Kesimal, H. Deveci, and F. Erdemir, "Paste backfill of high-sulphide mill tailings using alkali-activated blast furnace slag: effect of activator nature, concentration and slag properties," Minerals Engineering, vol. 83, pp. 117-127, 2015.

[11] E. Yilmaz, T. Belem, and M. Benzaazoua, "Specimen size effect on strength behavior of cemented paste backfills subjected to different placement conditions," Engineering Geology, vol. 185, pp. 52-62, 2015.

[12] E. Yilmaz, T. Belem, and M. Benzaazoua, "Effects of curing and stress conditions on hydromechanical, geotechnical and geochemical properties of cemented paste backfill," Engineering Geology, vol. 168, pp. 23-37, 2014.

[13] M. Fall, M. Benzaazoua, and E. G. Saa, "Mix proportioning of underground cemented tailings backfill," Tunnelling and Underground Space Technology, vol. 23, no. 1, pp. 80-90, 2008.

[14] M. Fall, T. Belem, S. Samb, and M. Benzaazoua, "Experimental characterization of the stress-strain behaviour of cemented paste backfill in compression," Journal of Materials Science, vol. 42, no. 11, pp. 3914-3922, 2007.

[15] N. Sivakugan, R. M. Rankine, K. J. Rankine, and K. S. Rankine, "Geotechnical considerations in mine backfilling in
Australia," Journal of Cleaner Production, vol. 14, no. 12-13, pp. 1168-1175, 2006.

[16] N. Sivakugan, K. Rankine, and R. Rankine, "Chapter 18 geotechnical aspects of hydraulic filling of underground mine stopes in Australia," in Elsevier Geo-Engineering Book Series, vol. 3, pp. 513-538, Elsevier, Amsterdam, Netherlands, 2005.

[17] R. M. Rankine, K. J. Rankine, N. Sivakugan, W. Karunasena, and M. L. Bloss, "A numerical analysis of the arching mechanism in paste fill during a complete mining sequence," in Computational Mechanics-New Frontiers for the New Millennium, pp. 461-466, Elsevier, Amsterdam, Netherlands, 2001.

[18] N. Sivakugan, K. Rankine, and R. Rankine, "Chapter 3-geotechnical aspects of hydraulic filling of Australian underground mine stopes A2-Indraratna, Buddhima," in Ground Improvement Case Histories, J. Chu and C. Rujikiatkamjorn, Eds., pp. 83-109, Butterworth-Heinemann, San Diego, CA, USA, 2015.

[19] Z. Aldhafeeri and M. Fall, "Time and damage induced changes in the chemical reactivity of cemented paste backfill," Journal of Environmental Chemical Engineering, vol. 4, no. 4, pp. 4038-4049, 2016.

[20] Z. Aldhafeeri and M. Fall, "Sulphate induced changes in the reactivity of cemented tailings backfill," International Journal of Mineral Processing, vol. 166, pp. 13-23, 2017.

[21] Z. Aldhafeeri, M. Fall, M. Pokharel, and Z. Pouramini, "Temperature dependence of the reactivity of cemented paste backfill," Applied Geochemistry, vol. 72, pp. 10-19, 2016.

[22] B. Ercikdi, G. Külekci, and T. Yılmaz, "Utilization of granulated marble wastes and waste bricks as mineral admixture in cemented paste backfill of sulphide-rich tailings," Construction and Building Materials, vol. 93, pp. 573-583, 2015.

[23] B. Koohestani, T. Belem, A. Koubaa, and B. Bussière, "Experimental investigation into the compressive strength development of cemented paste backfill containing nano-silica," Cement and Concrete Composites, vol. 72, pp. 180-189, 2016.

[24] B. Koohestani, A. Koubaa, T. Belem, B. Bussière, and H. Bouzahzah, "Experimental investigation of mechanical and microstructural properties of cemented paste backfill containing maple-wood filler," Construction and Building Materials, vol. 121, pp. 222-228, 2016.

[25] N. J. F. Koupouli, T. Belem, P. Rivard, and H. Effenguet, "Direct shear tests on cemented paste backfill-rock wall and cemented paste backfill-backfill interfaces," Journal of Rock Mechanics and Geotechnical Engineering, vol. 8, no. 4, pp. 472-479, 2016.

[26] D. Wu, Y. Zhang, and Y. Liu, "Mechanical performance and ultrasonic properties of cemented gangue backfill with admixture of fly ash," Ultrasonics, vol. 64, pp. 89-96, 2016.

[27] D. Wu, Y. Hou, T. Deng, Y. Chen, and X. Zhao, "Thermal, hydraulic and mechanical performances of cemented coal gangue-fly ash backfill," International Journal of Mineral Processing, vol. 162, pp. 12-18, 2017.

[28] D. Wu, Y. Zhang, R. Zhao, T. Deng, and Z. Zheng, "A coupled thermal-hydraulic-mechanical application for subway tunnel," Computers and Geotechnics, vol. 84, pp. 174-182, 2017.

[29] S. Yin, A. Wu, K. Hu, Y. Wang, and Y. Zhang, "The effect of solid components on the rheological and mechanical properties of cemented paste backfill," Minerals Engineering, vol. 35, pp. 61-66, 2012.

[30] R. Le and C. Lassonde, "Comparison of the material properties of in situ and laboratory prepared cemented paste backfill," in Proceedings of the 2002 Annual Conference, Vancouver, BC, Canada, 2002. 
[31] T. Belem, M. Benzaazoua, B. Bussière, and A. M. Dagenais, "Effect of settlement and drainage on strength development within mine paste backfill," in Proceedings of the 9th International Conference on Tailings and Mine Waste, pp. 139-148, Vail, CO, USA, January2002.

[32] B. D. Thompson, W. F. Bawden, and M. W. Grabinsky, "In situ measurements of cemented paste backfill at the Cayeli mine," Canadian Geotechnical Journal, vol. 49, no. 7, pp. 755-772, 2012.

[33] T. Belem, A. Harvey, R. Simon, and M. Aubertin, "Measurement and prediction of internal stresses in an underground opening during its filling with cemented fill," in Proceedings of the 5th International Symposium on Ground support in Mining and Underground Construction, Perth, Australia, September 2004.

[34] X. Zhang, J. Lin, J. Liu, F. Li, and Z. Pang, "Investigation of hydraulic-mechanical properties of paste backfill containing coal gangue-fly ash and its application in an underground coal mine," Energies, vol. 10, no. 9, p. 1309, 2017.

[35] S. C. Zhang, B. Shen, Y. Y. Li, and S. F. Zhou, "Modeling rock fracture propagation and water inrush mechanisms in underground coal mine," Geofluids, vol. 2019, Article ID 1796965, 15 pages, 2019.

[36] Q. Zhang, S. Wang, X. Ge, and H. Wang, "Modified MohrCoulomb strength criterion considering rock mass intrinsic material strength factorization," Mining Science and Technology (China), vol. 20, no. 5, pp. 701-706, 2010.

[37] R. A. Galindo, A. Serrano, and C. Olalla, "Ultimate bearing capacity of rock masses based on modified Mohr-Coulomb strength criterion," International Journal of Rock Mechanics and Mining Sciences, vol. 93, pp. 215-225, 2017. 